expressed or not. It must be borne in mind, however, that even if these changes occur in the first ten minutes or so after the termination of the second stage of labour it is best, in any case, to wait at least another ten minutes, unless some contra-indication such as hemorrhage is present, so as to allow the membranes ample time to become separated from the uterine wall. We cannot say at present with any certainty when the membranes are sufficiently separated to be expelled entire, but we know from clinical observations that if the expression of the placenta be practised from some 10 to 15 minutes after it has passed into the lower uterine segment the chance of the retention of any portions of the membranes is very small indeed.

The question we have now to consider is, What are we to do if we find that the changes in the uterus indicative of separation of the placenta do not occur? Shall we try expression or shall we wait? In the great majority of cases the rise in the level of the fundus of the uterus and the bulging of the lower uterine segment are easily recognisable, but in patients with a great deal of abdominal fat it may not be easy to determine whether these conditions are present or not. In such a case, and even in cases where the external signs of separation are not very evident, an attempt at expression may be made after the lapse of half an hour. Supposing such attempts repeated two or three times fail. is it correct treatment, in the absence of any severe h:morrhage or other indication, at once to remove the placenta with the hand? In view of the results obtained by the method of waiting for spontaneous expulsion of the placenta it is clearly not correct treatment. Manual removal of the placenta should not be practised in the absence of any urgent condition immediately recuiring it for at least from one and a half to two hours after the birth of the chidd. It is more especially in cases where the woman is exhausted by a difficult or pro. longed labour that ample time should be allowed to see if the placenta will not become separated spontaneously. A the end of from one and a half to two hours further attempts may be made at expression and if they fail manual removal should then be carried out. In cases treated in this way the number requiring manual removal of the placenta is very small indeed.

In 6800 labours in the Marburg clinique treated by Ahlfeld's ${ }^{16}$ method of waiting from one and a half hours to two bours before undertaking manual removal of the placenta the operation was performed in 53 cases, $0 \cdot 78$ per cent, or once in every 128 labours. In Olshausen's clinique where Credé's method is practised, Seyffarth ${ }^{17}$ has recorded during the years 1895 to 190179 cases of manual removal of the placenta in 9465 labours, 0.83 per cent., or 1 in 119 cases. In 10,387 confinements in the Dublin Lying-in Hospital recorded by Dr. Joseph Clarke ${ }^{i \gamma}$ in 1817 in only 21, or 1 in 494, was manual removal of the placenta required. At that time it was the custom in that hospital to wait from two to 24 hours after the delivery of the child for the expulsion of the placenta. These figures show how very rarely it is necessary to remove the placenta manually when a sufficient length of time is allowed for its spontaneous expulsion.

The reasons why we should avoid whenever possible the performance of this operation are at once evident when we remember that manual removal of the placenta is attended with a relatively high mortality. Of 12,000 cases of labour recorded by Rosenthal ${ }^{19}$ in Munich during the years 1890 to 1900 in $39,0.3$ per cent., or 1 in 309 cases, manual removal of the placenta was practised with a mortality of 13 per cent This compares very unfavourabl $\nabla$ with a death-rate amongst the same series of cases of 8.6 per cent. for Casarean section, $4 \cdot 8$ per cent. for version, and $4 \cdot 4$ per cent. for forceps deliveries. Further than this the morbidity of such cases is unduly high. Of the 39 cases of removal of the placenta in only one-third were the patients free from fever during the puerperium and in another third this period was attended with very marked disturbances. Hegar's ${ }^{20}$ statistics show a death-rate of 11 per cent. after manual removal of the placenta, $4 \cdot 7$ per cent. after the application of forceps, and $7 \cdot 7$ per cent. after version and excraction of the foetus.

16 Ahlfeld : Zentralblatt fiur Grnäkologie, No. 11, 1904, p. 345. 17 P. Seyffarth : Über Manuelle Losing von Placenta und Placentarresten nach reifer Geburt, Inaugural Dissertation, Berlin, 1901. $18 \mathrm{~J}$. Clarke: Transactions of the Medical Association, Dublin, 1817, p. 369 .

19 Rosenthal : Die Fälle von Künstlicher Lösung der Placenta 20 Hegar: Beiträge zur Geburtshu!fe und Gynäkologie, Iand i., I, 1898.
The risk of the introduction of septic material into the veins or lymphatics of the uterine wall is especially great because during the separation of an adherent placenta the fingers are brought into such immediate contact with these structures. It is probable that the more general use of sterilised rubber gloves whilst nerforming this operation will tend to reduce its dangers and to lower the mortality rate, and this is a precaution which should always be taken.

From this short and necessarily imperfect consideration of some of the points in the management of the third stage of labour, we may, I think, draw the following conclusions :1. The third stage of labour is hest treated by the employment of the Dablin or Credés method of expressing the placenta after a sufficient interval of time has been allowed to elapse for the placenta to become separated from the uterine wall. 2. The tendency to shorten this interval of time to much less than the 30 minutes recommended by Credé is attended with considerable risk to the mother 3 . It is, however, best to be guided by careful observation of the changes in the uterus indicating that separation of the placenta has or has not taken place rather than by any arbitrary period of time. 4. After the change in the level of the fundus of the uterus takes place. indicating that the separation of the placenta has occurred, from 10 to 15 minutes should still be allowed to elapse so as to diminish the risk of the retention of any portion of the placenta or membranes. 5. If there is reason to suppose that the placenta is adherent and a trial of expression fails no harm will result to the mother in the absence of any urgent indications if a period of from one and a half to two hours is spent in waiting for its spontaneous separation and expulsion. 6. Manual removal of the placenta should never be performed if it can be avoided, and it should not be practised in the absence of severe hremorrhage or any other indication for its immediate performance until from one and a half to two hours have elapsed since the birth of the child, and then only if further attempts at expression fail. 7. Retained portions of placenta and membranes should be removed at once, unless in the case of the membranes they are of quite small size. 8. In performing this operation it is a wise precaution to wear sterilised rubber gloves.

THE

INFLUENCE OF THE NERVOUS SYSTEM AND EXTERNAL TEMPERATURE UPON CERTAIN CIRCULATORY CHANGES CONCERNED IN THE ETIOLOGY OF CATARRH, ULCFR, AND SIMPLE DILATATION OF THE STOMACH, WITH SUGGESTIONS AS TO TREATMENT.

By ALFRED MANTLE, M.D. DURH., M.R.C.P. LOND., PHYSICIAX TO THE ROYAL HALIFAX IXYTRMARY.

For several years I have been paying attention to disturbances of the vaso-motor system and $I$ have observed a large number of cases of one kind and another in the wards and out-patient department of the Royal Halifax Infirmary. Whilst noticing the circulatory changes so commonly seen in the extremities I have been impressed with the frequency with which these symptoms are associated with others suggestive of circulatory disorders in one or other organs of the bodg. I have been struck with the fact that the bulk of patients under my care suffering with gastric disorder, whether ulcer or simple dilatation, are of a neurotic temperament and show marked circulatory changes in the extremities which have existed for a long period before the onset of the gastric symptoms or have appeared coincidently or subsequently to their development, and I have asked myself the question, Is there not a connexion between the gastric symptoms and those manifesting themselves in the extremities?

There is normally a very close relationship between the systemic and splanchnic circulation wbich includes the stomach and other viscera, and disturbances in the one sooner or later crtate dirturbances in the other and destroy that physiological balance which should exist. It is 
important to remember that some people are very susceptible to circulatory disorders and there is great significance in the remari, so frequently heard in the consulting-room, "I have always a poor circulation," which we may not have always appraised at its value. As already noticed, these patients are always more or less neurotic, and in such peripheral circulatory disturbances are common, but they may be only temporary and not so extensive as to alter appreciably the balance between the systemic, splanchnic, or other circulation, particularly if the earlier symptoms have been recognised and dealt with. But, on the other hand, if vaso-motor changes in the periphery are neglected and no treatment is adopted to remedy the same, which unfortunately is so often the case with the poor, there may develop functional symptoms pointing to a considerable alteration in the circulation of some organ or organs, and this may involve the stomach, particularly if carelessness occurs in diet. I know that coldness and cold extremities are mentioned as symptoms occurring with certain stomach diseases, but only as symptoms secondary to some gastric disorder. I now wish to deal with them as physical signs of an important disturbance of the circulation, aggravating and sometimes causing gastric disease. Sir Thomas Barlow has noted the appearance of stomach and other symptoms in two or three of his published cases of Raynaud's disease, which would seem to have been induced by peripheral changes affecting the splanchnic circulation. One of these was that of a woman who at the outset of the attack of cyanosis of the extremities had nausea and sometimes vomiting, showing a disturbance in the gastric circulation; and another case he records in a boy who not only had gastric symptoms but hæmoglobinuria with vaso-motor disturbances in the extremities. Abercrombie ${ }^{1}$ also reports the case of a boy who suffered pain in the stomach when the hands were cyanosed and the urine was normal, but subsequently hæmoglobinuria developed. Porter ${ }^{2}$ reported the case of a middle-aged woman who was liable to attacks of local syncope and asphyxia of the hands and feet, occurring three or four times a day and alternating with paroxysmal attacks of epigastric pain and vomiting, usually followed by slight jaundice. Albumin and bile were found in the urine after such a seizure but no blood or blood colouring matter was detected.

Causes of disturbance of the gastric circulation.-The gastric circulation may be disordered in three ways : (1) through the central nervous system; (2) by the irritation of food; and (3) by changes of external temperature. All of these causes may be combined in bringing about the circulatory change.

Mental causes : Exoitement and norry.-Functional disorder of the stomach as part of a general functional disturbance of the nervous system has been too little considered and we are too ready to blame some article of food for most of our gastric troubles before making any investigation into the condition of the nerrous system and circulation. Undoubtedly the majority of those who suffer with gastric disease give a history of worry and anxiety and many are very sensitive to temperature changes. Goodhart says ${ }^{3}$ : "If I were going to write a book upon indigestion I should first devote myself to a volume on disease of the nervous system." It is evident that the nervous disturbance mainly acts by altering the blood-supply of the stomach and Sidney Martin states: "Changes in the blood-supply and in the processes of innervation are of great importance in stomach disease." Any mental excitement or worry may cause or aggravate an attack of indigestion and this is probably through a deficiency of splanchnic control, which allows blood to yield to gravity and pass to the splanchnic area of the abdomen instead of the limbs. Leonard $\mathrm{Hill}^{5}$ has pointed out how vaso-motor constrictor control of the splanchnic area normally forms the resistance box of the circulation and prevents gravitation. A few days ago a lady who was consulting me stated that whenever she had anxiety she felt some chilliness of the body, with discomfort and pain in the stomach; and another patient of the same type told me that she always felt worry referred to the stomach, with an abnormal feeling of coldness of the body and extremities. The late Dr. H. G. Sutton ${ }^{6}$ wrote : "Unde

Archives of Pediatrics, 1886, vol. iii., p. 567

THF JANCET, April 27th, 1889, p. 841

Diseases of the Stomach, p. 156

5 Allbutt's System, vol. vii, p. 250

Lectures on Pathology, p. 280 . extreme mental depression and harass the stomach, like the face and other surfaces, may become congested and undergo catarrhal inflammation and the vessels become so full that hæmorrhage may result." These cases all point to the great influence of the mind over the circulation of the stomach-a fact demonstrated by Pawlow in his experiments upon gastric secretion.

Dietetic errors are ohiefly manifest in the nerrous.-The gastric circulation, as we know, is also disturbed locally by the ingestion and irritation of some article of food or beverage, and if the irritation continues not only is there a local but a general disturbance of the circulation. When dietetic errors produce so called dyspeptic symptoms it is chiefly in those of a nervous temperament, excepting some who abuse themselves with alcohol, tobacco, \&c., for it is extraordinary how tolerant the stomach is and what it will digest without producing any symptoms of disturbed function when the nervous system is normal, and some of the most robust will say, "I can eat anything." I suggest, then, that it is in the nervous that indiscretion in diet shows itself most frequently, and then by locally disturbing the innervation of the stomach and producing reflexes which excite a hyper-sensitive vaso-motor centre. If dietetic errors be persisted in, the more or less continuous irritation causes an increasing tendency for blood to be drawn to the splanchnic area and leave the periphery, hence the development of symptoms of coldness of the body and extremities in chronic gastric disturbance. I emphasise this as being an important development, for any increase in the amount of blood in the splanchnic area tends to aggravate the already congested stomach, and other patho. logical changes such as superficial ulceration of the mucous membrane may follow, or a gradual dilatation of the organ, through loss of motility. With the poor, and particularly young women in the northern manufacturing towns, the abuse of tea is very common, and I believe more detrimental to the stomach than almost anything else, for tea is the popular beverage but is too frequently allowed to infuse for an indefinite time before being used.

Grave oirculatory disturbances excited by gastric irritation.-We sometimes see local irritation in the stomach cause very acute gastritis or catarrh and the general circulation may become so quickly and completely disorganised as to border on collapse. This condition generally occurs in those who have previously shown functional disturbance of the stomach and who have taken some article of food which has acted as an irritant. I have seen several of such cases explored for perforation which has not been found. In one case a young woman in the Royal Halifax Infirmary who had been under observation for gastric catarrh ate some strawberries. Shortly afterwards symptoms of collapse developed and were of so serious a character that Dr. Priestley Leech, under whose care she was, explored the abdomen in order to find out if perforation had taken place, but found none. In another case under my colleague, Dr. W. Lockwood, a woman who for several years had suffered with gastric symptoms, after partaking of some pickled cabbage was seized with pain in the stomach, followed by the development of a condition of collapse of such a serious character that after consultation with his colleagues it was thought to be probably due to gastric perforation and an exploratory operation was deemed desirable. Pye-Smith ${ }^{7}$ of Sheffield has published similar cases in which he explored the abdomen in these circum. stances expecting to find gastric perforation, but nothing was found to account for the symptoms. This extreme condition would appear to be brought about by irritation in the stomach provoking afferent impulses to the vaso-motor centres and causing such a dilatation of the vessels of the splanchnic area and fall of blood pressure that blood which ought to be in the periphery is stagnated there, and is sometimes confused with that due to ptomaine poisoning. It also demonstrates the gravity of a great displacement of blood to the splanchnic area. Stewart ${ }^{8}$ has said: "Not a drop of blood may be lost from the body and yet death may occur from hæmorrhage into the abdominal cavity, into the stomach, or intestines. A man may bleed to death into his own blood-vessels."

The infuence of externat temperature upon the gastric circulation.-Having shown that the stomach circulation may be disturbed by mental influences and by local irritation

\footnotetext{
Quarterly Medical Journal, 1901.
} 
of food, we must now consider what the influence of external temperature is upon the gastric circulation, for this has an important bearing upon treatment. The effect of temperature upon the individual varies considerably and whilst the majority do not feel change of temperature in any marked degree there are others much affected by it, the more nervous and particularly women being most sensitive. Some feel both extremes of temperature, but by far the largest class feel the cold abnormally; they seem intended for warmth and express themselves as only comfortable in warm or mild weather. The feeling of coldness may be limited to the extremities or particular parts of the body, or may be more or less general. With different instruments $^{9}$ I have taken the blood pressure in many of these cases and $I$ find that cold tends to raise the arterial pressure whilst the venous pressure is lowered, and though these changes may be only temporary, yet with frequent narrowing of the systemic area by exposure, and a diminished tone in the splanchnic arterioles, there is a disposition in some to loading of the splanchnic veins and accumulation of blood in the splanchnic reservoir. Others feel heat abnormally and seem designated for cold weather, and in them the arterial pressure is found low and the venous high, but these do not suffer so much with gastric troubles as with cutaneous and other disturbances. Nearly all vaso-motor disturbances in the periphery are affected by changes of external temperature and may be observed there, but these changes, if considerable, cannot take place without others occurring internally, of a more or less compensatory character.

The influence of a cold atmosphere upon the cutaneous circulation is in some very rapid and circulatory changes may take place in the coronary, pulmonary, splanchnic, kidney, or other circulation in those exposed, but there can be no rule as to which organ will be chiefly affected. Confining our attention to the stomach, we must confess that the effect of external temperature upon gastric disorders has been too little regarded, important as it is. We see some who are liable to functional disturbance of the stomach markedly affected by changes of temperature; for instance, the change of temperature during the early hours of the morning may cause the patient to wake with coldness of the extremities and gastric pain, or the difference between the temperature of a warm room and one which is cold may be sufficient to cause discomfort in the stomach, whilst in others a cold east wind very often causes pain, accompanied or not with flatulence and nausea. The same people are sensitive to cold water and cannot take a cold bath, which has a similar effect upon them. In these cases the blood, driven from the skin by contraction of its vessels, chiefly affects the splanchnic area and is a similar displacement to that which normally takes place during the act of digestion, though the excitant in the latter instance is reflex from the stomach and is excited by food, whilst in the former it is the stimulus of a cold temperature acting upon the vaso-motor centres which brings about the changed condition of the cutaneous circulation. When food is introduced into the stomach an enormous amount of blood is drawn to this part for purposes of digestion and there is a correspondingly small amount in other areas, such as the skin, and in the healthy a feeling of chilliness is not infrequently experienced after a full meal (Stewart).

similar circulatory change brought about by exposure acting upon the sensitive nervous apparatus of the periphery is abnormal and if constantly repeated, as in the case with those much exposed in gaining a livelihood, may produce a chronic congestion and blood stasis, which if prolonged will become serious to the organs chiefly concerned.

The bulk of my hospital patients with gastric disease are young women employed in mills Such an occupation tends greatly to aggravate any predisposition to circulatory changes. Rising very early in the morning and frequently with little or no food and that least suited to their condition, not too well clad, they face the weather, what ever it may be, and if working in a heated mill (more especially a cotton mill) are subjected throughout the day to extremes of heat and cold. The domestic servant represents another class undergoing a similar kind of experience and should she have the same 9 I have used Oliver's hremadynamometer and Martin's modification
of the Riva-Roci instrument, but the compressed air manometer with of the Rlva-Rocci instrument, but the compressed air manometer with by Dr. George Olliver I have found particularly sensitive as well as reliable. tendency to vaso-motor disturbances her early morning duties may expose the sensitive peripheral vessels to sudden chills and the organ or organs most susceptible suffer. A servant maid who had gastric perforation and who was successfully operated upon by a colleague stated that she had learned by experience not to use cold $\mathrm{w}$ ater in her domestic work, the immersion of the hands causing so much discomfort and pain in the epigastrium; whilst another, under my care for hæmatemesis, described the epigastric pain as always coming. on in the early morning when cleaning steps in a very exposed and cold position. Comparing the life of these workers with one in a different social position, having every comfort but with the same predisposition to circulatory changes, can we wonder that there is such a marked class distinction in those usually suffering with gastric ulcer, for undoubtedly it is more commonly seen among the poor?

Seasonal influence upon gastric disorders. - We must admit that gastric cases are most prevalent during the cold season of the year. I have made observations for several years on this point which conclusively prove that functional disturbances of the stomach and liver are more commonly met with during the first and last quarter of the year. Lauder Brunton ${ }^{10}$ says, "Many people who are perfectly well during the dry weather begin to suffer from dyspepsia as soon as the weather becomes cold and damp." Robert Hutchison ${ }^{11}$ has recently remarked, "It is in the spring of the year that most dyspeptics are loudest in their complaints." Risien Russell, ${ }^{12}$ writing on gastric tetany, which in the adult is frequently the result of gastric dilatation, says that exposure to cold will produce the tonic contraction of the extremities, and according to Gunbrecht nearly three-fourths of the cases of tetany occur between the months of January and March. Eustace Smith $^{13}$ has recently pointed to the influence of chill in producing acute gastric catarrh and disturbance of the liver in children.

Splanchnic congestion from malaria and burns of body.-. There are other conditions which bring about an active congestion of the splanchnic area and we must consider if they are in any way connected with changes in the stomach amongst other organs. In the cold stage of malarial fever we have a big displacement of blood from the skin to the internal organs which often causes nausea, vomiting, and intense headache, the pulse being small in volume and the urine increased in quantity. In severe cases there is hæmorrhage from the mucous membrane of the stomach and bowel, also hæmoglobinuria or hæmaturia. Rokitanski attributed some of his cases of gastric ulcer to malaria and if we consider the congested condition of the mucous membrane of the stomach in the remittent form when there is probably a condition of splanchnic stasis there is some reason for associating the two diseases, and English authors have spoken of malaria as a cause of ulcer of the stomach. In burns of the body, in the stages of shock and collapse, there is a great determination of blood to the splanchnic and other areas, and ulceration of the duodenum and stomach is seen. The fact of the ulceration in burns taking place about the tenth day has been thought by some to point to septic emboli as the cause, but it is difficult to understand why the emboli in the circulation should generally select the duodenum for their point of attack. In injury to the skin it is not uncommon to have catarrbal inflammation of the mucous membrane of the upper part of the small intestine and stomach and not infrequently mucous and submucous hæmorrhages, and in three cases of duodenal ulceration examined by Fenwick he found evidence of them being secondary to hæmorrhage. It is then probable that ulceration of the intestine in burns follows upon small hæmorrhages in the congested mucous membrane, the congestion being the result of shock and an abnormal collection of blood in the splanchnic area.

Splanchnic stasis considered in the etiology of gastric ulcer.

-In considering the etiology of gastric ulcer we have not sufficiently recognised the important part the nervous system plays in gastric disorders and still less have we considered any circulatory changes in the condition except those in the stomach itself. We must fully recognise that branches of the coliac arteries which supply the stomach with blood carry with them nerves from the coliac plexus, giving a direct connexion with the great sympathetic plexuses in the abdomen. We then realise that any disturbance of these

10 Allbutt's System, vol. iii., p. 199

Brit. Med, Jour., vol. ii., p. 1383, 1905

13 Brit. Med. Jour., vol. i., 190e, p. 308. 
nerves may affect the circulation, not only locally but generally. "There is much to be considered about the solar and coeliac plexuses in relation to simple ulcer of the stomach and to nervous distress in the stomach region." 14

I have shown that a predisposition to gastric disorders is commonly associated with a functional disturbance of the nervous system and tendency to circulatory changes and I have pointed out that the stomach disturbances may arise through prolonged mental influences, irritation of food, or by changes of external temperature, for facts show that there may be a plus amount of blood in the splanchnic area involving the stomach with a diminution in the periphery under each of these influences. I have also shown that with a similar blood displacement in malaria and burns the stomach and bowel are frequently involved. What I now wish to point out is that such a condition of congestion is liable to be accompanied by hæmorrhages into the mucous membrane and sometimes into the submucous tissue which may be the starting points of ulceration. In the congested stomach mechanically produced in cardiac disease and portal obstruction hæemorrhages are found, and the same are common whenever congestion of the stomach is severe. Fenwick ${ }^{75}$ believes that there is congestion of the stomach at the menstrual periods, accompanied by hæmorrhages, and if these are unusually deep and extensive, he thinks that they may be the origin of acute gastric ulceration. But whatever the amount of congestion may be at this time, it is of a temporary character and less likely to be associated with hæmorrhages into the mucous or submucous tissue than the congestion of splanchnic stasis which, once established, may last for weeks or months. It easily follows that some of these hæmorrhages may result in abrasions or erosions which may be prevented from healing by the irritation of the gastric secretion, so commonly hyper-acid, and proof is not wanting that the gastric juice does act in an irritating way upon the injured mucous membrane. For when the cesophagus is seen ulcerated it is generally in that part which comes in contact with the regurgitated acid chyme. The same applies to the duodenum, which is less often "ulcerated in the lower end, where the chyme is alkaline, than near the pylorus, where the chyme is acid. We conclude, then, that acute ulceration may have its origin in a hæmorrhagic erosion, acted upon by the acid secretion of the stomach, and that this may become chronic if steps are not taken to relieve the congestion which has caused the hremorrhage and to prevent its repetition. In the etiology of many cases of ulcer several causes operate together, some being immediate and some remote.

Oral sepsis is a secondary cause. - Oral sepsis has been urged as a cause of ulcer and may be a powerful agent in infecting any hæmorrhagic erosion of the mucous membrane and in furthering ulcerative changes. But I cannot think that micro-organisms from the mouth will infect the mucous membrane of the stomach, unless there is some breach of surface, such as the superficial ulceration seen in chronic catarrh and congestion following hæmorrhages, for we see very carious teeth and no gastric ulcer, and, on the other hand, gastric ulcer with no signs whatever of oral sepsis. Oral sepsis, however, may prove to be an important complication of any circulatory disturbance involving the stomach.

Thrombosis and anemia.-Virchow thought gastric uleers originate through embolism or degenerative changes in the arteries arresting the circulation, a condition of infarct being produced and necrotic changes in the mucous membrane. 'The slowness of the circulation in the stomach in a state of congestion may favour thrombosis of its vessels but it has been seldom seen post mortem with gastric ulcer, whilst hæmorrhages and hæmorrhagic erosions are common. Degeneration of vessels is met with at a later period of life than that in which a majority of cases of gastric ulcer occur but may be the cause of thrombosis and ulcer in older subjects. Anæmia has been looked upon as an indirect cause of gastric ulcer and undoubtedly we see it in acute as well as in chronic ulcer but in the latter condition it must be considered as partly due to malnutrition. I am convinced, however, that true anæmia in gastric ulcer is not so commonly seen as what George Oliver ${ }^{16}$ describes as a quasi-anæemic appearance, the result of splanchnic drain, and in the latter condition he says: "The examination of the blood does not. as a rule, support the theory of anæmia, for the

14 H. G. Sutton: Leetures on Pathology, p. 283 16 Blood and Blood Pressure, p. 269 . proportion of the hæmoglobin and of the red discs fall well within the normal limits of variation. The pallid appearance is not due to an impaired quality but to a reduced amount of blood in the vessels."

Hematemesis of splanchnic stasis, - I have no doubt that many cases of hæmatemesis are due to congestion of the mucous membrane of the stomach, part of a splanchnic stasis, no ulceration being present, and Mayo Robson has described two cases he explored and in each a general rather than a local bleeding was found. Bertrand Dawson ${ }^{17}$ has named these cases of homorrhagic gastralgia and has pointed out the difficulties of diagnosis from ulcer. Hale White ${ }^{13}$ describes similar cases which he says are not due to ulceration and occur in women chiefly between 30 and 40 years of age. He makes no mention of any circulatory disturbances having been noticed and $I$ think that probably many of them are due to congestion, part of a splanchnic stasis. As pointed out by Essex Wynter, ${ }^{12} 80$ per cent. of cases of brematemesis occur in young women with hardly a death and hrematemesis in the wards of a hospital is a rare occurrence. It is the rest, warmth, and freedom from exposure there which, by relieving the congested condition of the stomach, account for this.

Simple or atonic dilatation and splanchnic stasis.-In taking part in the discussion upon the causes of dilatation of the stomach introduced by Professor T. Clifford Allbutt at Manchester in 1902, I pointed out the disturbance of the circulation commonly seen with gastric dilatation. More extended observations have shown very great peripheral changes with stomach dilatation, lividity of the extremities having been observed in some cases. Dilatation may follow upon gastric catarrh, the catarrh being due to some irritant in the stomach, or it may be part of a general neurasthenia produced by mental anxiety, whilst in others the changes in the peripheral circulation are consequent upon exposure and if there is a very sensitive vaso-motor system it would appear to be the disturbing factor. In all the result is the same there is too much blood in the splanchnic area and too little in the periphery. Loss of motility in the stomach is succeeded by dilatation and as a result of food fermentation toxins are generated which sometimes create other disturbances shown by vertigo, tetany, and palpitation.

Ciroulatory changes in $3 ?$ cases of gastrio and duodenal uloer operated upon. - The diagnosis of gastric ulcer is not always easy, but in order to satisfy myself that the circulatory disturbances described as found with gastric disorders are present in cases of ulceration, I have investigated this point in 31 cases in which gastric or duodenal ulceration has been proved by operation. I am indebted to my surgical colleagues at the Royal Halifax Infirmary, past and oresent, and to $\mathrm{Mr}$ H. Littlewood and Mr. B. G. A. Moynihan of the Leeds Infirmary, who have allowed me to investigate some of their cases operated upon for perforation or gastro-enterostomr. These consisted of 24 cases of ulcer of the stomach in young females which had perforated, one of gastric ulcer in a male and anotber in a female, for which gastro-enterostomy had been performed, and six duodenal ulcers in men. In 18 of the 26 gastric cases, the patients described themselves as having been very sensitive to cold all their lives with a poor peripheral circulation; seven stated that the coldness of the extremities and general sensitiveness to cold temperatures bad come subsequently to the gastric disorder and that they were normal before thus suggesting a stomach origin of the circulatory disturbance. In one case of gastric perforation the patient expressed herself as having been always warm. Of the six duodenal cases two expressed themselves as having been always abnormally sensitive to cold and dyspeptic symptoms, but four stated they bad been naturally warm until the onset of the gastric trouble. Practically all would be described as of a nervous temperament and nearly all gave a history of worry, which would affect the gastric circulation through another source already described. Brinton, years ago, pointed out that there was a history of worry in nearly all of his cases of ulcer. The other striking feature in this analysis is the fact that 20 of the 31 cases gave a history of having had a weak peripheral circulation for the greater part of their life and of being very easily influenced by external temperature. For the most part they were working people, worried and much

$1 \pi$ Brit. Med. Jour., vol. ii., 1902, p. 1709.

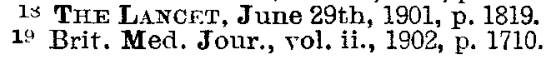


exposed in gaining their livelihood : the very life to augment any loss of circulatory balance.

Suggestions in treatment: The importance of external narmth.-The general lines of treatment of gastric disturbances are familiar to all, but one important consideration as an outcome of this inquiry demands our special attention. We must not confine ourselves to the stomach only but remember the disturbance of the nervous system and circulation and not only give rest to the stomach but obtain the same for body and mind if possible. If there is ulceration or dilatation this is best accomplished by placing the patient in bed and by doing so we assist the next point in treatment, which is the restoration of the disturbed circulation. To accomplish this heat must be applied, by the use of hot bottles, to the feet and other cold parts. A large amount of blood is thus drawn from the splanchnic area to the limbs and skin surface and a better circulation soon relieves the splanchnic stasis which has produced the congested stomach. As the general condition improves and the circulation becomes more normal, exercise is most helpful. Most patients find out the necessity of warm clothing and apply it to the most sensitive parts and this must be encouraged by us. In the case of gastric dilatation in addition to warmth, washing out of the stomach and anti-fermentative treatment are important. The importance of recognising symptoms of failure of the peripheral circulation while occurring during the course of gastric disease cannot be over-estimated when we remember that more blood to the congested mucous membrane of the stomach must be detrimental to the healing of an ulcer. For six or seven years I have treated all cases of gastric disturbance, including chronic catarrh, ulcer, and dilatation, in my wards in this way and $I$ always give the strictest instructions as to the importance of warmth in similar cases when in the outpatient department. The same treatment must be adopted to counteract the circulatory disorder in the case of chronic ulcer. But when this and other treatment have failed, and being satisfied that the ulcer is chronic, the operation of gastro-enterostomy should be seriously considered, for only then is the ulcer given an opportunity of healing and with the removal of the source of irritation the circulatory disturbance may disappear. Other local treatment to correct the tendency to splanchnic stasis must be directed to the splanchnic area by means of a well-applied broad bandage or an abdominal belt, which by exercising pressure tends to express blood from this part into the general circulation. We must also fully recognise that there is a serious loss of nerve tone and endeavour to reinstate it.

In conclusion, I strongly urge that inasmuch as nearly all or many peripheral vaso-motor disturbances of the circulation may be associated with circulatory disturbance of some organ or organs they should receive more attention at our hands, and that their cause should be investigated and treatment adopted with due appreciation of the value of warmth in remedying the same. We use very active treatment to restore the circulation in the condition of collapse and shock, but we may be too slow in recognising and treating the lesser disturbances of the peripheral circulation concerned not only in gastric but in other disorders. I only wish that the poor who suffer thus could be tanght how much comfort they might obtain and at what a trifling cost by the use of the hot bottle. The mouth should be examined for carious teeth or other sources of infection in all cases of gastric disorder inasmuch, as already stated, any superficial ulceration of the stomach may become infected by organisms from the mouth and develop into deeper ulceration. It is equally important to treat anæemia if present, for its existence will hinder any reparative process.

Harrogate.

Association of Medical Men Receiving ResiDENT PATIENIS.-The annual general meeting of this association was held on March 30th at the offices of the British Medical Association. There was a good attendance of members. In the absence of the President, Dr. Walsh, through illness, the chair was taken by Dr. H. Forbes Winslow, Tice-President. The minutes of the previous general meeting having been read and confirmed the honorary secretary read a statement of the affairs of the association which led to an interesting discussion, and was considered to be of a satisfactory nature. The membership is 93 , of whom 13 are life members. Dr. Walsh was unanimously re-elected President and the other officers having been reappointed the proceedings terminated with a vote of thanks to the executive.

\section{A CASE OF GLTOMA OF THE PONS.}

\section{By G. SCHORSTEIN, M.D. OxON., F.R.C.P. LOND.} PHXSICIAN TO THE LONDON HOSPITAL, ETC.; AND

A. J. WALTON, M.R.C.S. ENG., L.R.C.P. Lond, HOLSE PHYSICIAN TO THE LONDON HOSPITAT.

THE patient, a male, aged four years, was admitted to the London Hospital on Sept. 9th, 1905. For two months his mother noticed that he had a squint, the left eye turning inwards; since then he had gradually got weak on his legs and for the last two or three weeks she had noticed that his speech had got weaker and slurring. His previous health had always been good. There was no evidence of any congenital syphilis. On admission the patient was unable to walk, owing to paralysis of the legs. The right leg was somewat spastic and could not be moved. The left leg was only paralysed in the foot, movement occurring both in the hip and knee-joints. There was marked loss of power in the right arm, there being no grip, whilst the movements of the elbow and shoulder were only just present. The left arm was normal. There was definite paralysis of the left third nerve with internal strabismus, the secondary deviation being more marked than the primary. There was paralysis of the left side of the face, of the nerve type, the corrugator supercilii and orbicularis oculi muscles being both affected. The knee-jerks were increased, more especially those of the right side. Ankle clonus and Babinski's sign were not obtained. The ocular fundi were clear and there was some incontinence of urine of the reflex type, the bladder filling and emptying involuntarily.

On Sept. 20th the following note was made. "For the last two days there has been a good deal of difficulty in swallowing, but the palate moves well. The tongue is protruded to the right. The left arm and leg are now spastic, but not to so marked a degree as those on the right. The reflexes are also more marked on the right side. Since admission there have been several fits; during these the patient remains quite conscious but the muscles, and more especially those of the left side, twitch, so that the left arm and leg are continuously in action. The paralysis of the cranial nerves is unaltered. The fundi remain clear; temperatures taken on either side of the body show no marked differences." A diagn sis of a tumour in the left half of the pons was now made. On the 25th there was a slight fit, lasting for a few minutes only, during which both sides of the body twitched. The paralysis of the right arm had become rather more extensive. By the 27 th the con. dition was worse; there was now difficulty in taking food, the act of swallowing not being easily performed. There was some loss of power over the trunk muscles, so that the child was unable to sit up. There was some difficulty in getting the bowels open, so that purgatives had to be administered. On the 29th the child was in a much more helpless condition, being unable to move. It was now necessary to feed him with a nasal tube. The fits were more frequent, there having been three in the previous week; they were of the same nature as before. There was no incontinence. The following notes were taken as to the conditions of the fundi : "Right: the disc shows a well-defined and pigmented border except at the lower and inner side where the edge is illdefined and the disc somewhat swollen The vessels are not buried, there is no exudate of lymph along them, and there are no hæmorrhages. Left : this is similar to the right but the edge is not so well defined." In the hope that the tumour might be gummatous in nature three-grain doses of potassium iodide were given daily in a simple mixture.

On Oct. 3rd it was noted that the condition was gradually getting worse. The right arm was very weak so that articles could not be grasped. The right leg was spastic and the reflexes on that side were much increased. The condition of the left leg had not changed; the movements of the left arm were incoördinate. On the night of the 2 nd there was another fit which lasted five minutes. After that the right side of the palate did not move so well as the left on movement, the whole palate being drawn over to the left. The tongue was protruded to the left. At 7 P.M. on the 5 th there was a severe fit lasting for a quarter of an hour, during 